\title{
Vólvulo sigmoideo pediátrico por enfermedad de Chagas
}

\section{Pediatric sigmoid volvulus due to Chagas disease}

Víctor H. García-Orozco ${ }^{*}$, Daniel García-Sánchez², Juan F. López-Flores ${ }^{3}$ y Ricardo Sánchez-Mata

${ }^{1}$ Servicio de Cirugía General; ${ }^{2}$ Servicio de Cirugía Pediátrica; ${ }^{3}$ Banco de Leche Humana. Hospital Civil de Tepic Dr. Antonio González Guevara, Servicios de Salud de Nayarit, Tepic, Nayarit, México

\begin{abstract}
Resumen
La exposición y las infecciones por Trypanosoma cruzi ocupan el cuarto lugar entre las causas de pérdida de años de vida potenciales por enfermedades parasitarias e infecciosas. Se describe el caso de un niño de 11 años, con cuadro de oclusión intestinal, intervenido quirúrgicamente con datos de vólvulo intestinal. La pieza quirúrgica se envió a histopatología, que reportó megacolon chagásico. El rango de edad de presentación es un reto ante la falta de síntomas inespecíficos. No se cuenta con datos estadísticos pediátricos que definan la tripanosomiasis en estado latente o crónico, y estos niños serán diagnosticados en la etapa adulta por las alteraciones fisiopatológicas que presentarán.
\end{abstract}

PALABRAS CLAVE: Enfermedad de chagas. Megacolon. Oclusión intestinal. Tripanosomiasis. Vólvulo de sigmoides.

\begin{abstract}
Exposure and infections by Trypanosoma cruzi are the fourth cause of loss of potential life years between parasitic and infectious diseases. We describe the case of a 11-year-old patient with intestinal occlusion, surgically treated with intestinal volvulus, the surgical specimen is sent to histopathology reporting Chagasic megacolon. The age range of presentation is a challenge in the absence of nonspecific symptoms. There is no pediatric statistical data that define trypanosomiasis in a latent or chronic state and will be diagnosed in adult stages due to the physiopathological alterations that they will present.
\end{abstract}

KEY WORDS: Chagas disease. Megacolon. Intestinal occlusion. Trypanosomiasis. Sigmoid volvulus.

\section{Introducción}

La exposición y las infecciones por Trypanosoma cruzi ocupan el cuarto lugar entre las causas de pérdida de años de vida potenciales por enfermedades parasitarias e infecciosas ${ }^{1}$. La tripanosomiasis americana, también conocida como enfermedad de Chagas, es una infección parasitaria transmitida por vectores hematófagos ${ }^{2}$ con presentación clínica aguda y una forma crónica silenciosa. Según la Organización
Mundial de la Salud, existen entre 16 y 18 millones de personas infectadas en el mundo, de las cuales la mayoría se encuentran en territorio latinoamericano, y solo el $1 \%$ recibe un diagnóstico adecuado y un tratamiento completo. En 2015 se reportaron en México 1095 casos nuevos de enfermedad de Chagas, de los cuales $6(0.5 \%)$ fueron en menores de 1 año y $13(1.2 \%)$ en el grupo de edad de 10 a 14 años ${ }^{3}$. Además, de 5463 casos de esta enfermedad estudiados entre 2000 y 2012, solo 171 (3.1\%) fueron casos crónicos con síntomas ${ }^{4}$. En Nayarit, durante 2016 se reportaron 18

\section{Correspondencia:}

*Víctor H. García-Orozco

Privada Regina, 913, Interior 11

Col. Santa Cecilia

Fecha de recepción: 25-05-2018

C.P. 63089 , Tepic, Nayarit, México

E-mail: zamadaj@ hotmail.com
Fecha de aceptación: 11-07-2018 DOI:10.24875/CIRU.18000478
Cir Cir. 2019;87:568-571

Contents available at PubMed www.cirugiaycirujanos.com 
casos nuevos de enfermedad de Chagas, en edades de 20 a 64 años, con el mayor porcentaje (61.1\%) en el grupo de 25 a 44 años, con una tasa de incidencia de 1.4 por cada 100,000 habitantes $^{5}$. Una de las principales complicaciones de la forma crónica de la enfermedad de Chagas es el desarrollo del megacolon chagásico, el cual produce alteraciones en el sistema neurosensorial del colon. En el desarrollo de volvulaciones intestinales secundarias a megacolon se incluyen alteraciones funcionales y mecánicas de dicho órgano, como son déficit de vitaminas, adherencias y procesos inflamatorios crónicos ${ }^{6}$.

\section{Presentación del caso}

Se recibe en urgencias pediátricas del Hospital Civil Dr. Antonio González Guevara de la ciudad de Tepic, Nayarit, México, a un varón de 11 años de edad, con padecimiento de 3 días de evolución, caracterizado por distención abdominal, dolor difuso y ausencia de evacuaciones y posteriormente incapacidad para canalizar gases, y con vómitos de forma intermitente con contenido gastrobiliar. A su llegada, se encuentra dolor abdominal difuso, náuseas, distención abdominal generalizada e incapacidad para canalización de gases y evacuación. En la exploración física se observa facies de dolor, mal estado de hidratación, abdomen distendido, ausencia de peristalsis, timpanismo generalizado, resistencia involuntaria y dolor, y ámpula rectal colapsada con restos de materia fecal sin datos de impactación. Se inician soluciones cristaloides, analgésico, sonda vesical y sonda nasogástrica, por la cual se obtiene material gastrobiliar en cantidad aproximada de $400 \mathrm{ml}$. Los análisis paraclínicos reportan trombocitosis de $402,000 \mathrm{cel} / \mathrm{mm}^{3}$, leucocitosis de $18,260 \mathrm{cel} / \mathrm{mm}^{3}$ con neutrofilia y electrolitos con hipopotasemia de $2.5 \mathrm{mmol} / \mathrm{l}$. Las radiografías de abdomen muestran distención generalizada de asas intestinales, edema de pared intestinal e imagen compatible con un vólvulo intestinal (Fig. 1). Se solicita turno quirúrgico y, previo consentimiento informado de los padres, se somete a laparotomía exploradora en la que se encuentran los siguientes hallazgos: distención generalizada de asas, volvulación doble a nivel de sigmoides con lesión mesentérica secundaria (Fig. 2), escaso líquido inflamatorio peritoneal de aproximadamente $50 \mathrm{cc}$, sin datos de perforación intestinal ni contaminación intestinal de la cavidad abdominal. Se realiza sigmoidectomía con anastomosis primaria término-terminal de colon descendente-recto y colocación de drenaje Penrose. Se envía la pieza

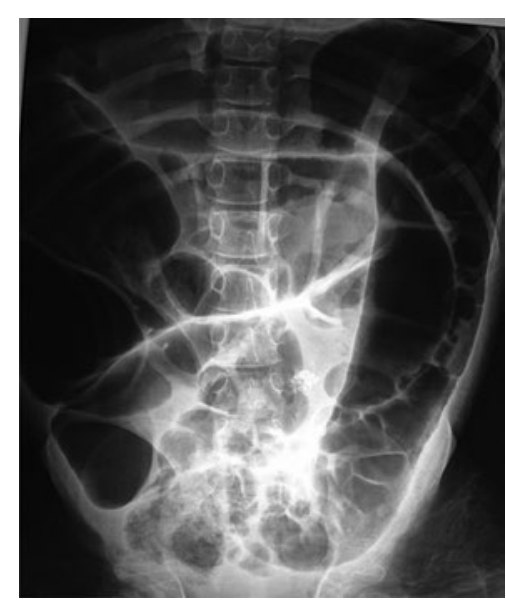

Figura 1. Radiografía simple de abdomen. Distensión generalizada de asas intestinales con edema secundario y ausencia de aire distal.

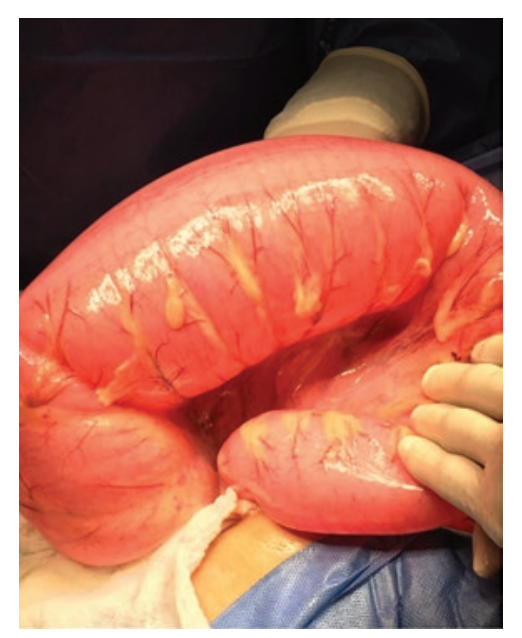

Figura 2. Hallazgos transoperatorios. Vólvulo de sigmoides con distensión proximal de asas intestinales y lesión mesentérica.

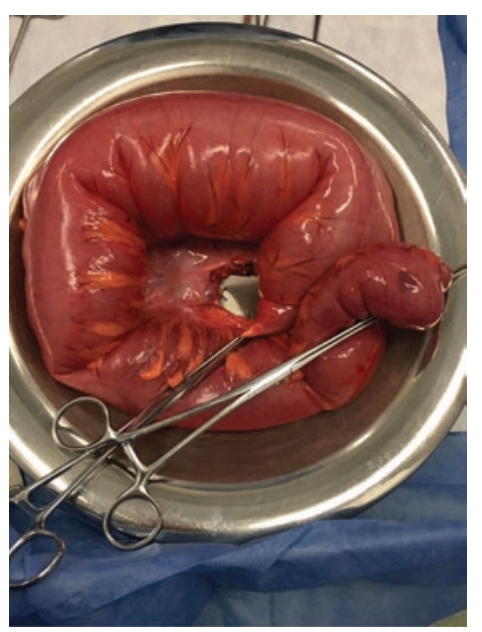

Figura 3. Resección de sigmoides para envío a estudio histopatológico.

quirúrgica para su estudio histopatológico (Fig. 3). El paciente continúa su manejo posquirúrgico con soluciones para requerimientos, antibiótico, analgésicos, 
control de líquidos y movilización asistida por familiares, y cursa con evolución hacia la mejoría, manteniéndose afebril. Cinco días después, el paciente presenta salida de material intestinal a través del Penrose y dolor abdominal localizado en el hueco pélvico y la fosa ilíaca izquierda, por lo que se realiza una nueva intervención en la que se encuentran distención de asas intestinales con migración del epiplón hacia la fosa ilíaca izquierda y anastomosis con dehiscencia del $50 \%$ con salida de material intestinal contenida en la fosa ilíaca, por lo que se desmantela la anastomosis y se realizan colostomía y cierre del recto en bolsa de Hartmann. Tras una buena evolución es egresado para seguimiento en consulta externa. El estudio histopatológico refiere datos macroscópicos de intestino grueso de $85 \times 11 \mathrm{~cm}$ con dilatación completa. Al corte se observa una mucosa sin pliegues, congestiva, con bordes libres. El diagnóstico final es de megacolon por enfermedad de Chagas. Se realiza notificación al servicio de epidemiología y se envía el paciente para seguimiento por pediatría, infectología pediátrica y medicina preventiva.

\section{Discusión del caso y revisión bibliográfica}

Pese a que el vector se conoce desde el siglo xiv, los primeros informes de endemia corresponden al periodo poscolombino, debido a los desplazamientos humanos que condicionaron el contacto directo del vector con el huésped. En 1909, el Dr. Carlos Chagas comunicó el descubrimiento de la nueva tripanosomiasis humana, y en 1911 obtuvo el primer registro de enfermedad chagásica congénita. En 1949, Aldao, en Venezuela, describió el hallazgo de T. cruzi en sangre periférica de un recién nacido de 2 días de vida². La transmisión madre-hijo de T. cruzi puede ocurrir en cualquier etapa del desarrollo embrionario y fetal, sin estar exento de infección ninguno de los periodos del desarrollo. Sin embargo, para que se produzca la infección, debe encontrarse en periodo de parasitemia 7 . La forma congénita de la enfermedad chagásica tiene dos formas clínicas de presentación: asintomática, que corresponde a la mayoría de los casos, y asintomática, que puede manifestarse de forma precoz o tardía, según aparezcan los síntomas antes o después de los 30 días de vida extrauterina, siendo la taquicardia un signo clínico que denota posibilidad de miocarditis, que cuando se presenta suele ser persistente'.

En los conocidos como megasíndromes destacan el megaesófago y el megacolon chagásico. El primero suele presentarse antes de los 40 años de edad y se relaciona con la destrucción progresiva de las neuronas parasimpáticas que condicionan dilatación en diferentes grados y posterior elongación, con hipertrofia de las capas musculares y paraqueratosis mucosa, con áreas de inflamación crónica, que evoluciona a la pérdida progresiva de la coordinación motora y la capacidad contráctil, produciendo disfagia como síntoma principal. El megacolon suele presentarse con disfunción motora de diferentes segmentos del colon, siendo mayor en el sigmoides y el recto, condicionado por la denervación parasimpática intramural, que lleva progresivamente a la dilatación. La expresión clínica es la constipación crónica y progresiva, que se posterga debido a la falta de sospecha y el tratamiento sintomático. Una de las complicaciones principales de la dilatación es el desarrollo de vólvulos ${ }^{8}$.

La tripanosomiasis es un problema de salud en Latinoamérica ${ }^{9}$. En México, durante 2017 se reportaron 733 casos de enfermedad de Chagas, de los cuales $173(23.6 \%)$ corresponden a la fase aguda y $560(76.4 \%)$ a la fase crónica. Esta enfermedad predomina en los hombres, reportándose para este sexo $476(64.9 \%)$ casos y en mujeres 257 (35.1\%). En el Estado de Nayarit se acumularon siete casos nuevos de enfermedad de Chagas, lo que representa el $0.9 \%$ del total de casos del país ${ }^{10}$. Los cambios fisiopatológicos de la enfermedad conllevan alteraciones en la calidad de vida y, en algunos casos, ponen en riesgo la funcionalidad o la vida misma, siendo una de las principales causas de incapacidad y pérdida de años productivos. Requiere abordaje quirúrgico de urgencia cuando se presentan complicaciones como las volvulaciones intestinales, permitiendo resolver la urgencia y previniendo nuevos cuadros de oclusiones y vólvulos pese a no ser un tratamiento curativo ${ }^{11}$. Entre las condiciones que dificultan el diagnóstico y el manejo adecuado de la enfermedad se encuentran la falta de sospecha clínica y la facilidad de transmisión a través de un vector ampliamente distribuido en numerosas zonas de México ${ }^{12}$. Además, la falta de tamizaje en las mujeres en edad reproductiva o como control prenatal predispone a que la enfermedad de Chagas pueda ser adquirida de modo intrauterino y pasar desapercibida durante el periodo neonatal o infantil.

En este caso, la única sintomatología asociada fue estreñimiento crónico. El vólvulo de sigmoides es una patología relativamente infrecuente en pacientes de edad pediátrica. Al recibir los resultados histopatológicos compatibles con enfermedad de Chagas, se desconocen el tiempo de evolución y las complicaciones agregadas al momento de la atención urgente. La 
literatura médica actual define como manejo quirúrgico la resección de vólvulo con posibilidad de realizar anastomosis primaria o derivación intestinal y posterior reinstalación del tránsito intestinal. Es importante destacar que, debido a la presencia de megacolon chagásico en un paciente pediátrico, debemos pensar en la cronicidad del padecimiento y la posibilidad de haber sido adquirido de manera congénita, pasando desapercibido hasta el momento de la atención de urgencia.

México cuenta con un programa institucional de acción específica para la prevención y el control de la enfermedad de Chagas $^{4}$, con el cual se pretende disminuir la transmisión domiciliaria y realizar una detección oportuna de casos neonatales. Entre los problemas que persisten se encuentra el subregistro de casos debido a la falta de síntomas, y no se cuenta con un tamizaje sistemático para las mujeres embarazadas de zonas endémicas. Es esencial, ante un paciente en edad pediátrica en quien se detecte un megasíndrome digestivo no diagnosticado previamente, tener la sospecha de tripanosomiasis y solicitar estudios complementarios para el diagnóstico.

\section{Conclusiones}

Al encontrarnos en zonas endémicas de $T$. cruzi es crucial tener conocimiento sobre los medios de contagio y las características clínicas que pueden presentar los pacientes de acuerdo con la etapa en que se encuentre la enfermedad. Es importante destacar que no se cuenta con cifras epidemiológicas fidedignas que permitan contemplar un panorama exacto sobre la incidencia y la prevalencia de dicha enfermedad. De igual manera, es difícil realizar una estimación sobre el número de pacientes pediátricos que están cursando con tripanosomiasis en estado latente o crónico y que serán diagnosticados en etapas adultas; esto resulta importante por el posible sesgo que pudiera presentarse en el diagnóstico de la tripanosomiasis congénita. Por ello, ante pacientes que presenten sintomatología vaga y que, como en este caso, tengan alteraciones morfológicas con megalias orgánicas de origen no especificado al momento del hallazgo, deben realizarse estudios complementarios para descartar causas infecciosas, como la mencionada en esta publicación.

\section{Agradecimientos}

Los autores agradecen al personal que labora en el Hospital Civil de Tepic Dr. Antonio González
Guevara por su interés en la atención de calidad de los pacientes, así como por las facilidades para la recopilación de información correspondiente al presente artículo.

\section{Conflicto de intereses}

Los autores declaran que no existen conflictos de intereses entre los proponentes y los participantes en este trabajo.

\section{Responsabilidades éticas}

Protección de personas y animales. Los autores declaran que no se han realizado experimentos en seres humanos o animales para esta investigación.

Confidencialidad de los datos. Los autores declaran que han seguido los protocolos de sus centros de trabajo sobre la publicación de datos de pacientes.

Derecho a la privacidad y consentimiento informado. Los autores han obtenido el consentimiento informado de los pacientes y/o sujetos referidos en el artículo. Este documento obre en poder del autor de correspondencia.

\section{Bibliografía}

1. Paredes R. Prevalencia de Chagas congénito en recién nacidos del "Hospital Materno Infantil Poconas". En: Ramos M, Serrudo J, editores. Ciencias de la salud T-I Handbook. Bolivia: ECORFAN; 2014. p. 189-218.

2. Mansilla $M$, Rocha $M$, Sarubbi M. Chagas congénito. Presentación de un caso clínico y revisión bibliográfica. Rev Hosp Mat Inf Ramon Sardá. 1999;18:29-35.

3. Secretaría de Salud. Distribución de casos nuevos de enfermedad por grupos de edad. Estados Unidos Mexicanos 2015. México, D.F.: SUIVE Dirección General de Epidemiología; 2015.

4. Secretaria de Salud. Prevención y control de la enfermedad de Chagas, programa sectorial de salud 2013-2018. México, D.F.: Secretaría de Salud; 2014.

5. Secretaría de Salud. Distribución de casos nuevos de enfermedad por grupos de edad. Estados Unidos Mexicanos 2016. México, D.F.: SUIVE Dirección General de Epidemiología; 2016.

6. Frisancho O. Dolicomegacolon andino y vólvulos intestinales de altura. Rev Gastroenterol Perú. 2008;28:248-57.

7. Moya P, Basso B, Moretti E. Enfermedad de Chagas congénita. Aspectos epidemiológicos, clínicos, diagnósticos y terapéuticos. Experiencia de 30 años de seguimiento. Rev Soc Bras Med Trop. 2005;38(Suppl 11):33-40.

8. Werner Apt B, Heitmann I, Jercic M, Jofré L, Muñoz P, Noemí I, et al. Enfermedad de Chagas en el adulto, la infancia y la adolescencia. Rev Chil Infect. 2008;25:194-9.

9. Cucunubá Z, Valencia C, Puerta C, Sosa S, Torrico F, Cortés J, et al. Primer consenso colombiano sobre Chagas congénito y orientación clínica a mujeres en edad fértil con diagnóstico de Chagas. Infectio. 2014; (3):3-16.

10. Secretaría de Salud. Dirección General de Epidemiología. Boletín Epidemiológico del Sistema Nacional de Vigilancia Epidemiológica. Sistema Único de Información. Semana Epidemiológica No. 52, 2017. Disponible en: https://www.gob.mx/cms/uploads/attachment/file/285868/sem52.pdf.

11. Santos R, Meyer B, Féres O, Ribeiro J. Surgical treatment of Chagas megacolon. Critical analysis of outcome in operative methods. Act Cir Bras. 2008;23(Suppl 1):83-92.

12. Schmunis G. La tripanosomiasis americana como problema de salud pública. En: La enfermedad de Chagas y el sistema nervioso. Washington: OPS; 1994. Publicación científica 547. p. 3-29. 